\title{
Biological Lattice Gauge Theory as Modeling of Quantum Neural Networks
}

\author{
Yi-Fang Chang \\ Department of Physics, Yunnan University, Kunming 650091, China
}

\begin{abstract}
Based on quantum biology and biological gauge field theory, we propose the biological lattice gauge theory as modeling of quantum neural networks. This method applies completely the same lattice theory in quantum field, but, whose two anomaly problems may just describe the double helical structure of DNA and violated chiral symmetry in biology. Further, we discuss the model of Neural Networks (NN) and the quantum neutral networks, which are related with biological loop quantum theory. Finally, we research some possible developments on described methods of networks by the extensive graph theory and their new mathematical forms.
\end{abstract}

Keywords: biology, neurobiology, modeling, quantum mechanics, lattice gauge theory, network, graph theory.

\section{Introduction}

Biological systems are all very complex systems. Their descriptions apply usually some simplified methods of modeling from the well-known Hodgkin-Huxley model to modeling of spiking-bursting neural behavior using two-dimensional map [1], spontaneous emergence of modularity in a model of evolving individuals [2], collective signaling behavior in a networked oscillator model [3]. Based on the neural synergetics, we proposed Lorenz model of brain [4]. Contrarily, Gorbachev and Abramova applied a modified neuro-fuzzy model to the trajectory of world economic and technological development [5].

Based on the inseparability and correlativity of the biological systems, we proposed the nonlinear whole biology and four basic hypotheses [6]. Based on the extensive quantum theory we proposed the extensive quantum biology [7]. Assume that basic quantum elements of DNA are A-T and G-C, so DNA may apply the extensive quantum biology [8]. Further, the Schrödinger equation with the linear potential may become the Bessel equation. Its solutions are Bessel functions, and may form the double helical structure of DNA in three dimensional space [9]. In this paper we propose the biological lattice gauge theory as modeling of the quantum neutral networks, and new described forms of networks are researched.

\section{Biological Lattice Gauge Theory}

Quantum biology is well-known theory. For example, neurobiology applies widely quantum mechanics. Tarlaci proved we need quantum physics for cognitive neuroscience [10], and researched the probabilistic quantum thinking and obtained experimental results that are of basic significance in the fields of neuroscience and of psychology [11]. Erol researched basics and concise relations between Schrödinger wave equation and consciousness/mind [12]. We researched the possible applications of the Yang-Mills gauge theory in biology [6,13]. In particular, we discussed the SU(2) gauge theory of DNA, and corresponding equations and their some solutions [8]. We combined both aspects, and propose biological lattice gauge theory, which applies completely the same lattice gauge theory in quantum field. It is also the extensive quantum biology [7].

Wilson proposed lattice gauge theory, in which space-time is replaced by a discrete set of points [14]. Then Kogut, et al., discussed the theoretical Hamiltonian formulation, and so on [15]. This theory gives qualitative nonperturbative information concerning QCD, therefore, it becomes an important quantum field theory $[16,17]$.

In lattice gauge theory the simplest lattice is defined with equal lattice spacing $a$. When the limit $a \rightarrow 0$, it reduces to the usual Yang-Mills gauge theory, i.e., it is the discrete gauge field theory, and there is an effective largest momentum of order $1 / a$ as an ultraviolet cutoff [17]. Two neighboring sites of the lattice denote $(n, n+\mu)$. A member of SU(3) is [16]:

$$
U(n, n+\mu)=\exp \left[\operatorname{iag} \frac{\lambda^{a}}{2} A_{\mu}^{a}(n)\right] .
$$

Here $g$ is the coupling constant, $\lambda^{a}$ is the generator of $\mathrm{SU}(\mathrm{N})$, and $A_{\mu}^{a}(n)$ is the gauge field.

The scalars on the lattice must make the substitution: 


$$
\partial_{\mu} \phi \rightarrow \frac{1}{a}\left(\phi_{n+\mu}-\phi_{n}\right)
$$

Such the scalar action becomes:

$$
\mathrm{S}=\sum_{n}\left[\frac{a^{2}}{2} \sum_{\mu=1}^{4}\left(\phi_{n+\mu}-\phi_{n}\right)^{2}+a^{4}\left(\frac{m^{2}}{2} \phi_{n}^{2}+\frac{\lambda}{4 !} \phi_{n}^{4}\right)\right] .
$$

Here $\phi_{n}$ replace its Fourier transform $\phi(k)$, and insert the Fourier expansion of $\phi_{n}$ into the free action of the scalar field on the lattice. On the lattice, the propagator is generated [16]:

$$
k^{2}+m^{2} \rightarrow m^{2}+\sum_{\mu} \frac{4}{a^{2}} \sin ^{2}\left(a k_{\mu} / 2\right)
$$

Therefore, the properties of the expression are examined by a formula [16]:

$$
\frac{1}{a^{2}} \sin ^{2}\left(a k_{\mu}\right)+m^{2}
$$

Eq.(4) and (5) are similar to biological helical structures. The lattice gauge theory may derive two anomaly problems [16]: 1) There is an unphysical doubling for each space-time dimension. But, it seems to correspond just to the double helical structure of DNA. 2) Another problem is related to the anomaly violated chiral symmetry. But, it is widely natural character that the chiral symmetry is violated in biology. Therefore, the biological lattice gauge theory should be cable to describe the double helical structure of DNA and violated chiral symmetry in biology. Moreover, the lattice gauge theory describes the confinement of quarks, which corresponds also to the biological inseparability.

\section{Quantum Neural Networks}

Watts and Strogatz [18] researched complex networks, and networks of coupled dynamical systems have been used to modeling of biological oscillators, neural networks, genetic control networks and many other self-organizing systems. They explored simple models of networks that can be tuned through this middle ground: regular networks 'rewired' to introduce increasing amounts of disorder, and found that these systems can be highly clustered, like regular lattices, yet have small characteristic path lengths, like random graphs. This is namely 'small-world' networks [18,19]. Complex networks show some topological statistical properties, and scale-free [20], i.e., they have exactly or approximately the distribution of power function, and group structures [21-25], and corresponding network evolutional model. It is related with the topological thermodynamics, in which Kirchhoff law and Tellegen theorem may be applied to neurobiology. If Kirchhoff current law is violated, it will show brain infarction.

Hopfield [26,27] proposed a type of neural networks model, whose basic nonlinear equations are:

$$
C_{i} \frac{d u_{i}(t)}{d t}=-\frac{u_{i}(t)}{R_{i}}+\sum_{j=1}^{N} T_{i j} g_{j}\left(u_{j}(t)\right)+I_{i}
$$

Christopher discussed bishop, neural networks for pattern recognition [28]. Wolfram found that cellular automata have four different results: stable state, period states, chaos and complex state [29]. Further, there are also quasiperiod and random states. We discuss the quantum neutral network, which may be related with biological loop quantum theory [6].

Network includes random network and scale-free network [30]. General bionetwork is not random networks, and form often network clusters, which are described by the clustering coefficient, and form "small-world" networks with the shortest path length [18]. Bionetworks may construct network motifs with feed-forward, bi-fan, bi-parallel and three chains forms [31,32], and modularity [33], etc.

A main tool of networks is statistics physics, which is related with the average field and the renormalization theory. Ravasz, et al., discussed the statistical description of complex network [34,35]. In phase transition, a phenomenological rule of order parameter is:

$$
\xi \propto\left|t-t_{c}\right|^{\beta}
$$

It is a nonlinear formula, and $\beta$ is fractal dimension. The scale-free network has stronger robustness, and obeys also the power law [22]:

$$
p(k) \sim k^{-n}
$$

It is the degree distribution formula with a fractal dimension $D=-n$. The scale-free and clustering in 
bionetworks coexist, and emerge the new hierarchical networks, whose formations [34] are some classical fractals, for example, Eq.(8). Further, the fractal dimension may be developed the complex dimension in both aspects of mathematics and physics [36-38]:

$$
D_{z}=D+i T
$$

When the complex dimension is combined with relativity, whose dimensions are three real spaces and one imaginary time, it expresses a change of the fractal dimension with time or energy, etc. It exists widely in biology [39]. We discussed their existence in the fractal's description of meteorology, seismology, medicine and the structure of particle, etc [36,38,39].

It is well-known that the neurobiological system is a very complex nonlinear system. Much neuron constructs the neural networks (NN), which are origin of neural system. Their models may be continuous or discrete. The pan-brain network combines the pan-brain level and the pan-brain relation. It includes complex macrocircuit and mirocircuit. These may be applied to neuron, neurobiology, and constitute their theoretical models, and research their functions and mechanism.

A connection model of Artificial Neural Networks (ANNs) is:

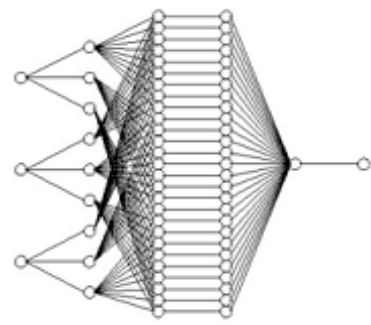

Fig. 1. A connection model of ANNs

There is the perception of neural networks. They may be self-adapt and self-organization, which corresponds to learning. Information may be storage and memory, may be control and learning. A three-layer connected model of $\mathrm{NN}$ is:

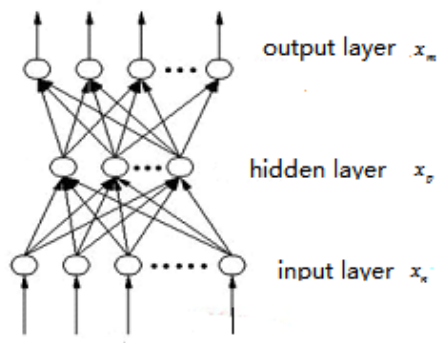

Fig. 2. A three-layer connected model of NN

In this model input layer $x_{n}$ has $n$ neurons, hidden layer $x_{p}$ has $p$ neurons and output layer $x_{m}$ has $m$ neurons. While $\mathrm{x}$ and $\mathrm{y}$ are not direct connection.

Barabasi and Albert [20] proposed the average field equations of evolutionary model on scale-free network:

$$
\frac{\partial k_{i}}{\partial t}=m k_{i} / \sum_{j=1}^{N-1} k_{j} .
$$

When time $\mathrm{t}$ is enough long, a solution is $k_{i}=m\left(t / t_{i}\right)^{\beta}$. When network increases with complete random, the average field equations are:

$$
\frac{\partial k_{i}}{\partial t}=\frac{m}{m_{0}+t-1} .
$$

Their solution is $p(k) \propto e^{-k / m}$, which is the same with Eq.(8)

Jeong, et al., [40] and Tanaka [41] researched metabolic networks, for example, amino acid biosynthesis, carbohydrate metabolism, lipid metabolism, nucleotide metabolism, etc. They have the small world property. Interacting networks of protein are also scale-free $[42,43]$. They and the channels in traditional Chinese medicine possess small world property and fractal structures.

If the relation between the clustering coefficient $\mathrm{C}(\mathrm{k})$ and scale $\mathrm{k}$ is $C(k) \propto k^{-\alpha}$, it will explain networks with the level structure. Networks possess clustering and hierarchical characters [33,34]. The social networks 
possess group structures [43-46,22,2]. Different sense systems of biology are namely different groups and communities. Newman proposed an important statistical parameter: assortativity $[47,48]$. It corresponds to the same sense system, etc.

Holme-Kim model of scale-free network has tunable clustering [45,49]. Networks can show dynamics with scale-free structures and nodes of networks [50-52], and form the coevolution network model [53,54], the adaptive network model [55], the self-organized network model [52], etc.

For structures of functional networks [56] the memory function is defined by the edge weight function $\mathrm{f}(\mathrm{e})$ in networks flow. When $f(e) \geq f\left(e_{c}\right)$, threshold value is namely long memory. It is related with initial conditions.

Entropy of degree distribution is defined by formula:

$$
H=-\sum_{k} P(k) \lg P(k)
$$

The maximum entropy corresponds to the uniform random networks, and the minimum entropy corresponds to the regular networks. It shows that degree distribution of small-world network method is analogy to degree distribution of random figure [19].

We proposed possible entropy decrease due to internal interactions in some isolated systems in biology, in which the neuroscience, the permeable membrane, the molecular motor, etc., are all some internal interactions. Further, a sufficient and necessary condition of entropy decrease is expressed quantitatively [57]. Entropy increase always will be unable to derive any complex systems. Moreover, we proposed quantitatively a universal entropy theory on evolution of any natural and social systems [8].

For synchronization model Kuramoto proposed a phase model with average field [58]. Pikovsky, et al., discussed universal synchronization [59]. Phase clustering and transition to phase synchronization in a large number of coupled nonlinear oscillators, and synchronization systems are researched [60,61]. By this method nonlinear oscillator may describe each object, and networks possess clustering structure [47,62]. Further, the networks model of synchronization may be constructed $[1,3]$ :

$$
\begin{aligned}
& x_{n+1}(i)=f\left[x_{n}(i), y_{n}(i)\right]+k[1-\delta(i, n)] \sum_{j \in S(i)} \delta\left(j, n-\tau\left(d_{i j}\right)\right), \\
& y_{n+1}(i)=y_{n}(i)-\mu\left(x_{n}(i)+1\right)+\mu \sigma, \quad\left(i=1,2, \ldots, N_{1}\right) .
\end{aligned}
$$

Here $\mathrm{k}$ is a key, which is bigger, and more synchronized. This is also a phenomenological field (as the thought field, religion field and Qi field, etc).

At recent Clauset, et al., researched hierarchical structure and the prediction of missing links in networks [63]. Newman and Clauset discussed structure and inference in annotated networks [64].

Bionetworks are related with biological topology, and both can be represented by matrix, where nodes are order and edges are values of elements. Points and edges form the complete incidence matrix. Biological networks are usually the non-directed graph, but conduction directions are the directed graph. We discussed biofield and some nonlinear theories in biology, researched chaos in biology and its application to cancer. Fractal and complex dimension in biology are searched. Nonlinear biothermodynamics and in which possible entropy decrease are investigated. We proposed the matrix representations of hypercycle theory. The quantum neural biology should be nonlinearity and quantization, and may relate to quantum chaos, quantized matrix, etc [39]. The matrix formulation is also a form of quantum mechanics, and is related with quantum biology and neural biology.

For structure of neuron, the weighted values, as neuron and element of matrix, may form various matrices. In this matrix the positive weighted values represent effect increase and negative values represent effect decrease. It should be four matrices (np, pn, pm, mp). They may be unified by one matrix:

$$
\begin{aligned}
& \left(H_{n p}+H_{p n}{ }^{\prime}\right)+\left(H_{p m}{ }^{\prime}+H_{m p}\right)=H_{2(n+m) p} . \\
& H_{2(n+m) p}=\left[\begin{array}{c}
H_{n p} \\
H_{p n}{ }^{\prime} \\
H_{p m}{ }^{\prime} \\
H_{m p}
\end{array}\right] .
\end{aligned}
$$




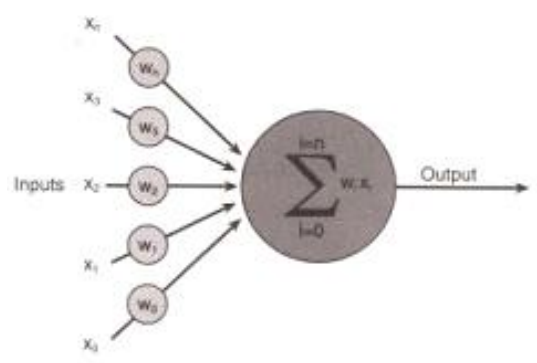

Fig. 3. Figure on inputs and output

General output is:

$$
X_{i}=\sum_{j=1}^{n} w_{j} x_{j}+s_{i}
$$

Here $s_{i}$ is the feedback information. From this forms the cycle mode. Its dynamic model is:

$$
\frac{d H}{d t}=F_{i p}
$$

It connects with Hopfield model.

Network dynamics researches the networks whose status changes in time [65-67]. It may be based on the principle of least action, and obtain network structures from selection principles [68-71]. We think that the basis of Chinese medicine is an old network theory, in which the channels are namely the special networks. The generalization of bionetworks is systems biology. Further, the quantum neural networks may combine the biological lattice gauge theory.

\section{New Research on Described Networks}

Hartwell, et al., [72] proposed that modern biology should develop new research methods, and investigate interactions among biological molecules, and corresponding network structures, dynamics and their function. The biological networks show the nonlinear whole biology [6].

One of the main mathematical tools on networks is graph theory. Bionetworks, neural networks and general networks may apply graph theory and the extensive field [38], etc. For example, the neural elements in neural networks correspond to nodes, and fields related complexity. Nodes and fields may form different levels and hierarchical networks. Here the importance of nodes may be represented by different centrality [73]. It can simplify the hierarchical networks [34]. In neural system there are various hierarchical interregional circuits, for example, local circuit, and microcircuit. They may be some biological lattices. Recently, Nadakuditi and Newman discussed graph spectra and the detectability of community structure in networks [74].

Based on the combination of the tree-field of graph and Feynman diagrams, we proposed a new development on graph theory, which includes five types of the basic elements: various solid lines, dotted lines, wavy lines, and vertices, fields (which are a set of legion small trees). Then, we researched their possible applications in physics and social sciences, etc [75]. The possible meanings of these elements are mathematically: solid lines represent connections with fixed values, dotted lines represent possible connections, wavy lines represent changed connections by function $\mathrm{f}(\mathrm{x})$, and fields represent complexity by sum formula $\sum_{\mathrm{i}=1}^{n} f_{i}$ and integral $\int f(x) d x$ or by product formula $\stackrel{i=1}{n}_{i=1}^{n}\left(f_{i}\right)$ in different levels, etc.

The new extensive graph may apply to various biological systems, which include more complex structures and joins. Bones are lines and tubes. Assume that cells correspond to fields, and various fibres correspond to different lines. The stomach is field, so intestine is line and 3-dimensional tube. We proposed the string theory of DNA and general biological string [8]. String is also a specific line for graph. Fig.3 is represented by lines, vertices and a field. 


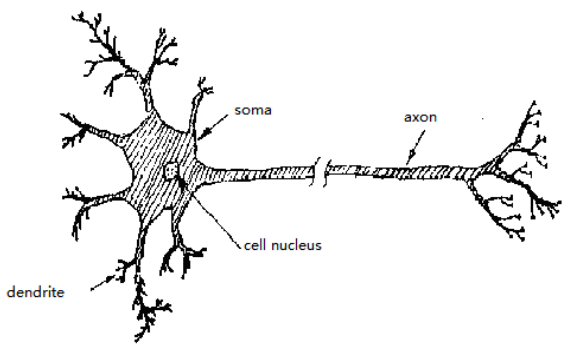

Fig. 5. Structure of neuron

In structure of neuron (Fig.5) the cell soma and neuron correspond to fields, and axon, dendrite and synapse correspond to various lines. Every cell body as all modeling Calabi-Yau manifold corresponds to superstring and higher dimension [76]. Further, we may research the Feynman rules of biology.

Primary model of neural transference may apply hydrodynamics and its three-dimensional shape. Assume $A \propto C^{D /(D+1)}$ [69]. For $\mathrm{D}=3$, so $A \propto C^{3 / 4}$. Theoretical explanation for DNA as a source of biophoton emission has been presented by Popp, et al [77]. According to the exciplex model the standing vibration waves (photons) in the DNA lattice have to provide the necessary conditions of allowed biophoton emission at all, and photons in DNA lattice are considered to originate from the stacking interaction in DNA staircase.

Further, this theory may combine the loop quantum gravity theory [6], and general relativity and twistor theory [78], etc.

\section{Conclusions}

Based on quantum biology and modeling by the lattice theory in quantum field, we propose the biological lattice gauge theory, whose two anomaly problems may just describe the double helical structure of DNA and violated chiral symmetry in biology. Further, we discuss the quantum neutral networks, which are related with biological loop quantum theory. Some possible developments on described methods of networks by the extensive graph theory and their new mathematical forms are researched.

In a word, the quantum neutral networks and general bionetworks may combine various theories and mathematical methods. It is possibly a direction of biology and neurobiology, and may be perfected and developed continually.

\section{References}

[1]N.F. Rulkov. Modeling of spiking-bursting neural behavior using two-dimensional map. Phys Rev E, 2002 : $65,041922$.

[2]J. Sun and M.W. Deem. Spontaneous emergence of modularity in a model of evolving individuals. Phys Rev Lett., 2007: 99,228107.

[3]Z. Liu and P.M. Hui. Collective signaling behavior in a networked oscillator model. Physica A. 2007: 383,714 .

[4]Yi-Fang Chang. A testable application of nonlinear whole neurobiology: Possible transformation among vision and other sensations. NeuroQuantology, 2013:11(3),399-404.

[5]S.V. Gorbachev and T.V. Abramova. A modified neuro-fuzzy model of the trajectory of world economic and technological development. Journal of Modeling and Optimization, 2017: 9(2), 100-111.

[6]Yi-Fang Chang. Nonlinear whole biology and loop quantum theory applied to biology. NeuroQuantology, 2012:10(2),90-197.

[7]Yi-Fang Chang. Extensive quantum biology, applications of nonlinear biology and nonlinear mechanism of memory. NeuroQuantology, 2012:10(2),183-189.

[8]Chang Yi-Fang. Extensive quantum theory of DNA and biological string. NeuroQuantology. 2014;12(3): 356-363.

[9]Yi-Fang Chang. Some solutions of extensive quantum equations in biology, formation of DNA and neurobiological entanglement. NeuroQuantology, 2015:13(3),304-309.

[10]S. Tarlac1. Why we need quantum physics for cognitive neuroscience. NeuroQuantology, 2010: 8(1),66-76.

[11]S. Tarlaci. On probabilistic quantum thinking. NeuroQuantology, 2010: 8(4),S1-2.

[12]M. Erol. Schrödinger wave equation and function: Basics and concise relations with consciousness/mind. NeuroQuantology, 2010: 8(1),101-109.

[13]Yi-Fang Chang. Neural synergetics, Lorenz model of brain, soliton-chaos double solutions and physical neurobiology. NeuroQuantology, 2013: 11(1),56-62.

[14]K.G. Wilson. Confinement of quarks. Phys Rev D, 1974: 10(8),2445-2459. 
[15]J.B. Kogut and L. Susskind. Hamiltonian formulation of Wilson's lattice gauge theories. Phys Rev D, 1975:11,395-408.

[16]M. Kako. Quantum Field Theory: A Modern Introduction. Oxford University Press. 1993.

[17]M. Srednicki. Quantum Field Theory. Oxford University Press. 2007.

[18]D.J. Watts and S.H. Strogatz. Collective dynamics of "small-world" networks. Nature, 1998: 339(6684), 440-442.

[19]A. Barrat and M. Weigt. On the properties of small-world network method. Eur Phys J, 2000: B13, 547-560.

[20]A.L. Barabasi and R. Albert. Emergence of scaling in random network. Science, 1999: 286,509-512.

[21]M. Girvan and M.E.J. Newman. Mixing patterns and community structure in networks. PNAS, 2002: $99,7821$.

[22]M.E.J. Newman and M. Girvan. Finding and evaluating community structure in networks. Phys Rev E, 2004: $69,026113$.

[23]M.E.J. Newman. Fast algorithm for detecting community structure in networks. Phys Rev E, 2004: $69,066133$.

[24]A. Clauset, M.E.J. Newman and C. Moore. Finding community structure in very large networks. Phys Rev E, 2004:70,066111.

[25]G. Palla, I. Derenyi, I. Farkas and T. Vicsek. Uncovering the overlapping community structure of complex networks in nature and society. Nature, 2005: 435, 814-818.

[26]J.J. Hopfield. Neural networks and physical systems with emergent collective computational abilities. Proc Natl Acad Sci USA, 1982:79,2554-2558.

[27]J.J. Hopfield. Neurons with graded response have collective computational properties like that of two-state neurons. Proc Natl Acad Sci USA, 1984:81,3088-3092.

[28]M. Christopher. Bishop, Neural Networks for Pattern Recognition. Oxford University Press. 1996.

[29]A. Ilachinski. Cellular Automata: A Discrete Universe. Singapore: World Scientific. 2001.

[30]A.L. Barabasi and E. Bonabeau. Scale-free networks. Scientific America, 2003: 288,60.

[31]R. Milo, S. Shen-Orr, S. Itzkovitz, N. Kashtan, D. Chklovskii and U. Alon. Network motifs: simple building blocks of complex networks. Science, 2002:298(5594),824-827.

[32]S. Shen-Orr, R. Milo, S. Mangan and U. Alon. Network motifs in the transcriptional regulation network of Escherichia coli. Nat Genet, 2002: 31(1),64-68.

[33]E. Ravasz, A.L. Somera, D.A. Mongru, Z.N. Oltvai and A.L. Barabasi. Hierarchical organization of modularity in metabolic networks. Science, 2002: 297(5586), 1551-1555.

[34]E. Ravasz and A.L. Barabasi. Hierarchical organization in complex networks. Phys Rev E, 2003: 67(2),026112.

[35]A.L. Barabasi. The architecture of complexity. IEEE Control Systems Magazine, 2007: 27(4),33-42.

[36]Yi-Fang Chang. The fractal model of particle, the complex dimension and its meaning. Exploration of Nature (China), 1988: 7(2),21-23.

[37]Yi-Fang Chang. New Research of Particle Physics and Relativity. Yunnan Science and Technology Press. 1989. Phys Abst, 1990: 93,1371.

[38]Fi-Fang Chang. New tree-field representations in graph theory, extension of Dirac extraction, differential test for series of positive terms, complex dimension and their applications. International Journal of Modern Mathematical Sciences, 2014:9(1),1-12.

[39]Yi-Fang Chang. Chaos, fractal in biology, biothermodynamics and matrix representation on hypercycle. NeuroQuantology, 2013:11(4),527-536.

[40]H. Jeong, B. Tombor, R. Albert, Z.N. Oltvai and A-L. Barabási. The large-scale organization of metabolic networks. Nature, 2000: 407,651-654.

[41]R. Tanaka. Scale-rich metabolic networks. Phys Rev Lett, 2005:94,168101.

[42]R. Albert and A-L. Barabási. Topology of evolving networks: local events and universality. Phys Rev Lett, 2000: 85,5234-5237.

[43]H. Jeong, S.P. Mason, A-L. Barabási and Z.N. Oltvai. Lethality and centrality in protein networks. Nature, 2001:411,41-42.

[44]E.M. Jin, M. Girvan and M.E.J. Newman. Structure of growing social networks. Phys Rev E, 2001: 64,046132.

[45]P. Holme, B.J. Kim, C.N. Yoon and S.K. Han. Attack vulnerability of complex networks. Phys Rev E, 2002 : $65,056109$.

[46]M.E.J. Newman and K. Park. Why social networks are different from other types of networks. Phys Rev E, 2003: 68,036122.

[47]M.E.J. Newman. Assortative mixing in networks. Phys Rev Lett, 2002:89,208701.

[48]M.E.J. Newman. Mixing patterns in networks. Phys Rev E, 2003:67, 026126.

[49]P. Holme and B.J. Kim. Growing scale-free networks with tunable clustering. Phys Rev E, 2004: 69,045101. 
[50]R. Albert, H. Jeong and A-L. Barabasi. Error and attack tolerance of complex networks. Nature, 2000: 406,378-382.

[51]S. Mossa, M. Barthelmy, H.E. Stanley and L.A.N. Amaral. Truncation of power law behavior in "scale-free" network models due to information filtering. Phys Rev Lett, 2002:88,138701.

[52]G. Bianconi and M. Marsili. Clogging and self-organized criticality in complex networks. Phys Rev E, 2004: 70,035105 .

[53]A. Barrat, M. Barthelemy and A. Vespignani. Weighted evolving networks: coupling topology and weight dynamics. Phys Rev Lett, 2004: 92,228701.

[54]J.M. Pacheco, A.T. Traulsen and M.A. Nowak. Coevolution of strategy and structure in complex networks with dynamical linking. Phys Rev Lett, 2006: 97,258103.

[55]T. Gross, C.D. D’Lima and B. Blasius. Epidemic dynamics on an adaptive network. Phys Rev Lett, 2006: 96,208701.

[56]V.M. Eguiluz, D.R. Chialvo, G.A. Cecchi, et al. Scale-free brain functional networks. Phys Rev Lett, 2005 : 94, 018102

[57]Yi-Fang Chang. Possible entropy decrease in biology and some new research of biothermodynamics. NeuroQuantology, 2013:11(2),189-196.

[58]Y. Kuramoto. Chemical Oscillations, Waves, and Turbulence. Berlin: Springer. 1984.

[59]A. Pikovsky, M. Rosenblum and J.J. Kurths. Synchronization----A Universal Concept in Nonlinear Science. Cambridge University Press. 2001.

[60]Z. Liu, Y.C. Lai and F.C. Hoppensteadt. Phase clustering and transition to phase synchronization in a large number of coupled nonlinear oscillators. Phys Rev E, 2001: 63, R055201.

[61]M. Barahona and L.M. Pecora. Synchronization systems. Phys Rev Lett, 2002: 89,054101.

[62]Z. Liu and B. Hu. Epidemic spreading in community networks. Europhys Lett, 2005: 72, 315-321.

[63]A. Clauset, C. Moore and M.E.J. Newman. Hierarchical structure and the prediction of missing links in networks. Nature, 2008: 453,98-101.

[64]M.E.J. Newman and A. Clauset. Structure and inference in annotated networks. Nature Communications, 2016: 7,11863.

[65]N.M. Luscombe, M.M. Babu, H. Yu, et al. Genomic analysis of regulatory network dynamics reveals large topological changes. Nature, 2004: 431(7006),308-312.

[66]L. Benda, N.L. Poff, D. Miller, et al. The network dynamics hypothesis: How channel networks structure. BioScience, 2004: 54(5),413-427.

[67]W.W. Powell and J. Owen-Smith. Network dynamics and field evolution: The growth of interorganizational collaboration in the life sciences. Ameri J Sociology, 2005: 110,901-975.

[68]J.R. Banavar, F. Colaiori, A. Flammini, A. Giacometti, A. Maritan and A. Rinaldo. Sculpting of a fractal river basin. Phys Rev Lett, 1997: 78(23), 4522-4525.

[69]J.R. Banavar, A. Maritan and A. Rinaldo. Size and form in efficient transportation networks. Nature, 1999: 399, 130-132.

[70]J.R. Banavar, F. Colaiori, A. Flammini, A. Maritan and A. Rinaldo. Topology of the fittest transportation network. Phys Rev Lett, 2000: 84(20), 4745.

[71]V. Colizza, J.R. Banavar, A. Maritan and A. Rinaldo. Network structures from selection principles. Phys Rev Lett, 2004: 92,198701.

[72]L.H. Hartwell, J.J. Hopfield, S. Leibler and A.W. Murray. From molecular to modular cell biology. Nature, 1999: 402,C47-52.

[73]T. Opsahl, F. Agneessens and J. Skvoretz. Node centrality in weighted networks: Generalizing degree and shortest paths. Social Networks, 2010:32(3),245-251.

[74]R.R. Nadakuditi and M.E.J. Newman. Graph spectra and the detectability of community structure in networks. Phys Rev Lett, 2012:108,188701.

[75]Yi-Fang Chang. New development on graph theory from Feynman diagram, and their applications in biology and other regions. International Journal of Modern Mathematical Sciences, 2014: 12(1),43-54.

[76]Yi-Fang Chang. Calabi-Yau manifolds in biology and biological string-brane theory. NeuroQuantology, 2015:13(4), 464-474.

[77]F.A. Popp, Q. Gu and K.H. Li. Biophoton emission: experimental background and theoretical approaches. Modern Phys Lett, 1994: B8,1269-1296.

[78]Yi-Fang Chang. Bioinformation and twistor theory in biology. NeuroQuantology, 2016:14(3), 589-596. 